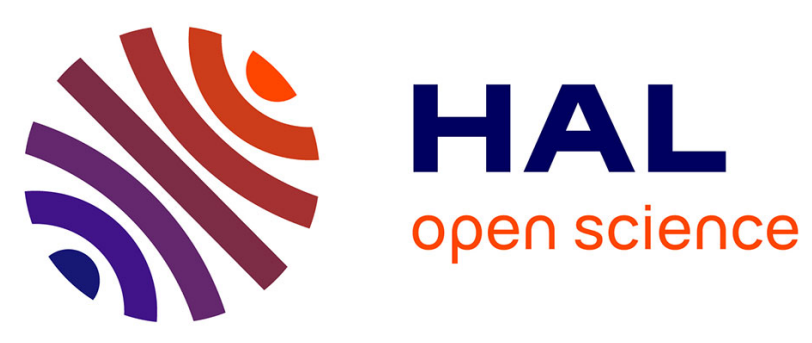

\title{
Breather mode in the many-electron dynamics of semiconductor quantum wells
}

F. Haas, Giovanni Manfredi, P.K. Shukla, Paul-Antoine Hervieux

\section{To cite this version:}

F. Haas, Giovanni Manfredi, P.K. Shukla, Paul-Antoine Hervieux. Breather mode in the manyelectron dynamics of semiconductor quantum wells. Physical Review B: Condensed Matter and Materials Physics (1998-2015), 2009, 80 (7), pp.073301-1-073301-4. 10.1103/PhysRevB.80.073301 . hal-00596639

\section{HAL Id: hal-00596639 \\ https://hal.science/hal-00596639}

Submitted on 30 May 2011

HAL is a multi-disciplinary open access archive for the deposit and dissemination of scientific research documents, whether they are published or not. The documents may come from teaching and research institutions in France or abroad, or from public or private research centers.
L'archive ouverte pluridisciplinaire HAL, est destinée au dépôt et à la diffusion de documents scientifiques de niveau recherche, publiés ou non, émanant des établissements d'enseignement et de recherche français ou étrangers, des laboratoires publics ou privés. 


\title{
Breather mode in the many-electron dynamics of semiconductor quantum wells
}

\author{
F. Haas, ${ }^{1}$ G. Manfredi, ${ }^{2}$ P. K. Shukla, ${ }^{1}$ and P.-A. Hervieux ${ }^{2}$ \\ ${ }^{1}$ Institut für Theoretische Physik IV, Ruhr-Universität Bochum, D-44780 Bochum, Germany \\ ${ }^{2}$ Institut de Physique et Chimie des Matériaux de Strasbourg, BP 43, F-67034 Strasbourg, France
}

(Received 22 July 2009; published 14 August 2009)

\begin{abstract}
We demonstrate the existence of a breather mode in the self-consistent electron dynamics of a semiconductor quantum well. A nonperturbative variational method based on quantum hydrodynamics is used to determine the salient features of the electron breather mode. Numerical simulations of the time-dependent Wigner-Poisson or Hartree equations are shown to be in excellent agreement with our analytical results. For asymmetric quantum wells, a signature of the breather mode is observed in the dipole response, which can be detected by standard optical means.
\end{abstract}

DOI: 10.1103/PhysRevB.80.073301

PACS number(s): 73.63.Hs, 73.43.Lp, 78.67.De

\section{INTRODUCTION}

The many-electron dynamics in nanoscale semiconductor devices, such as quantum wells and quantum dots, has recently attracted a great deal of interest, mainly in view of possible applications to the growing field of quantum computing. ${ }^{1}$ Particular attention has been devoted to intersubband transitions, which involve excitation frequencies of the order of the terahertz. ${ }^{2}$ On this time scale, various collective electronic modes can be excited. For instance, the electric dipole response is dominated by a strong resonance at the effective plasmon frequency. This resonance (known as the Kohn mode $)^{3}$ is characterized by rigid oscillations of the electron gas, which, for perfectly parabolic confinement, are decoupled from the internal degrees of freedom.

In this Brief Report, we show the existence of a new distinct resonance-a monopole or "breather" mode-which corresponds to coherent oscillations of the size of the electron gas around a self-consistent equilibrium. Breather modes have been described in many areas of physics, such as nuclear matter ${ }^{4,5}$ (where they are known as giant monopole resonances) and ultracold atom dynamics. ${ }^{6,7}$ In experiments on metallic nanoparticles, monopole oscillations of the ionic structure have been observed, which manifest themselves as slow modulations of the surface plasmon. ${ }^{8}$ However, to the best of our knowledge, previous investigations have not addressed the features of the breather mode in the selfconsistent dynamics of a confined electron gas. Although quantum wells constitute a typical instance of such confined systems, the present approach should equally apply to metal nanoparticles and carbon-based systems such as fullerenes.

\section{MODEL}

Because of the translational symmetry in the transverse plane, the problem reduces to a one-dimensional (1D) one in the $x$ direction. ${ }^{9,10}$ To model the electron dynamics, we use a self-consistent quantum hydrodynamic model (QHM) that was originally derived for quantum plasmas ${ }^{11,12}$ and metallic nanostructures. ${ }^{13}$ In the QHM, the evolution of the electron density $n(x, t)$ and mean velocity $u(x, t)$ is governed by the continuity and momentum equations

$$
\begin{gathered}
\frac{\partial n}{\partial t}+\frac{\partial}{\partial x}(n u)=0, \\
\frac{\partial u}{\partial t}+u \frac{\partial u}{\partial x}=-\frac{1}{m_{*} n} \frac{\partial P}{\partial x}-\frac{1}{m_{*}} \frac{\partial V_{\mathrm{eff}}}{\partial x}+\frac{\hbar^{2}}{2 m_{*}^{2}} \frac{\partial}{\partial x}\left(\frac{\partial_{x}^{2} \sqrt{n}}{\sqrt{n}}\right),
\end{gathered}
$$

where $m_{*}$ is the effective electron mass, $\hbar$ is the reduced Planck constant, $P(x, t)$ is the electron pressure, and $V_{\text {eff }}$ $=V_{\text {conf }}(x)+V_{H}(x, t)$ is the effective potential, which is composed of a confining and a Hartree term. The Hartree potential obeys the Poisson equation, namely $V_{H}^{\prime \prime}=-e^{2} n / \varepsilon$, where $e$ is the magnitude of the electron charge and $\varepsilon$ is the effective dielectric permeability of the material. The term proportional to $\hbar^{2}$ on the right-hand side of Eq. (2) represents the quantum force due to the so-called Bohm potential. ${ }^{14}$

The above QHM can be derived from the self-consistent Hartree equations ${ }^{12}$ — or equivalently from the phase-space Wigner-Poisson equations ${ }^{15}$ —in the limit of long wavelengths compared to the Thomas-Fermi screening length. For the sake of simplicity, we shall neglect exchange/correlation corrections and assume Boltzmann statistics, which is a reasonable approximation at moderate electron temperatures $T{ }^{9,16}$ We also stress that the $1 \mathrm{D}$ model relies on the separation of the transverse and longitudinal directions, which may be broken by collisional effects. However, such effects should not be dominant on the fast time scales considered here. $^{17}$

The pressure $P(x, t)$ in Eq. (2) must be related to the electron density $n$ via an equation of state (EOS) in order to close our system of electron fluid equations. We take a polytropic relation $P=\bar{n} k_{B} T(n / \bar{n})^{\gamma}$, where $k_{B}$ is the Boltzmann constant, $\gamma=3$ is the $1 \mathrm{D}$ polytropic exponent, and $\bar{n}$ is a mean electron density. For a homogeneous system (where $\bar{n}=n_{0}$ ), this EOS correctly reproduces the Bohm-Gross dispersion relation ${ }^{12}$ in quantum plasmas. For the inhomogeneous electron gas considered here, the choice of $\bar{n}$ is subtler and will be discussed later.

We assume a parabolic confinement, with $V_{\text {conf }}$ $=\frac{1}{2} \omega_{0}^{2} m_{*} x^{2}$, where the frequency $\omega_{0}$ can be related to a fictitious homogeneous positive charge of density $n_{0}$ via the relation $\omega_{0}=\left(e^{2} n_{0} / m_{*} \epsilon\right)^{1 / 2}$. We then normalize time to $\omega_{0}^{-1}$; space to $L_{0}=\left(k_{B} T / m_{*}\right)^{1 / 2} / \omega_{0}$; velocity to $L_{0} \omega_{0}$; energy to 
$k_{B} T$; and the electron number density to $n_{0}$. Quantum effects are measured by the dimensionless parameter $H=\hbar \omega_{0} / k_{B} T$.

We shall use typical parameters that are representative of semiconductor quantum wells: ${ }^{10}$ the effective electron mass and the effective dielectric permeability are, respectively, $m_{*}=0.067 m_{e}$ and $\varepsilon=13 \varepsilon_{0}$, the equilibrium density is $n_{0}$ $=4.7 \times 10^{22} \mathrm{~m}^{-3}$, and the filling fraction $\bar{n} / n_{0}=0.5$. These values yield an effective plasmon energy $\hbar \omega_{0}=8.62 \mathrm{meV}$, a characteristic length $L_{0}=16.2 \mathrm{~nm}$, a Fermi temperature $T_{F}$ $=51.8 \mathrm{~K}$, and a typical time scale $\omega_{0}^{-1}=76 \mathrm{fs}$. An electron temperature $T=200 \mathrm{~K}$ then corresponds to a value $H=0.5$.

\section{LAGRANGIAN APPROACH}

In order to derive a closed system of differential equations describing the breather mode, we fist express the quantum hydrodynamical equations in a Lagrangian formalism. We stress that this approach is not based on a perturbative expansion, and thus is not restricted to the linear regime. The Lagrangian density corresponding to the system of Eqs. (1) and (2) reads as (normalized units are used from now on)

$$
\begin{aligned}
\mathcal{L}= & \frac{1}{2}\left(\frac{\partial V_{H}}{\partial x}\right)^{2}-n V_{H}-n \frac{\partial \theta}{\partial t}-\int^{n} W\left(n^{\prime}\right) d n^{\prime}-\frac{1}{2}\left(n\left[\frac{\partial \theta}{\partial x}\right]^{2}\right. \\
& \left.+\frac{H^{2}}{4 n}\left[\frac{\partial n}{\partial x}\right]^{2}\right)-n V_{\text {conf }},
\end{aligned}
$$

where the independent fields are taken to be $n, \theta$, and $V_{H}$. The velocity field follows from the auxiliary function $\theta(x, t)$ through $u=\partial \theta / \partial x$. The quantity $W(n)$ in Eq. (3) originates from the pressure, $W \equiv \int \frac{d P}{d n^{\prime}} \frac{d n^{\prime}}{n^{\prime}}=(3 / 2)(n / \bar{n})^{2}$. Taking the variational derivatives of the action $S=\int \mathcal{L} d x d t$ with respect to $n, \theta$, and $V_{H}$, we obtain the Eqs. (1) and (2), as well as the Poisson equation for $V_{H}$.

The existence of a pertinent variational formalism can be used to derive approximate solutions via the time-dependent Rayleigh-Ritz trial-function method. ${ }^{18}$ For this purpose, we assume the electron density to have a GAUSSIAN profile

$$
n(x, t)=\frac{A}{\sigma} \exp \left[-\frac{(x-d)^{2}}{2 \sigma^{2}}\right],
$$

where $d(t)$ and $\sigma(t)$ are time-dependent functions that represent the center-of-mass (dipole) and the spatial dispersion of the electron gas, respectively. The constant $A=N / \sqrt{2 \pi}$, is related to the total number of electrons in the well, $N$ $=\int n d x$. The above Ansatz is a natural one, because for a negligible Hartree energy, $V_{\text {eff }}$ reduces to a harmonic oscillator potential.

The other fields to be inserted in the action functional are $\theta$ and $V_{H}$. The natural way to choose them is by requiring that the continuity and Poisson equations are automatically satisfied. The continuity equation is solved with $n$ given by Eq. (4) together with $u=\dot{d}+(\dot{\sigma} / \sigma) \xi$, which leads to $\theta$ $=(\dot{\sigma} / 2 \sigma) \xi^{2}+\dot{d} \xi$, where $\xi \equiv x-d$. An irrelevant gauge function was discarded in the calculation of $\theta$. The solution of the Poisson equation with a GAUSSIAN electron density is

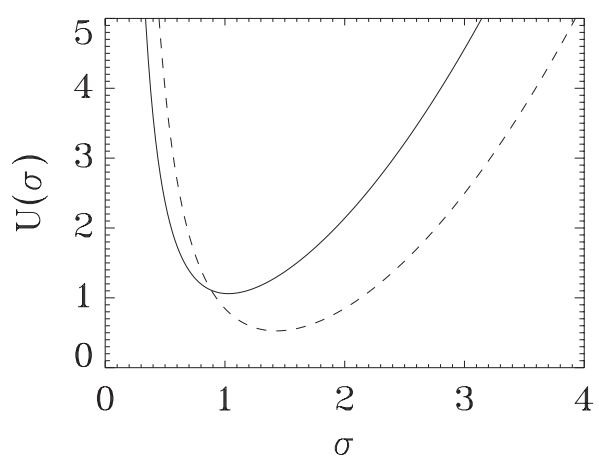

FIG. 1. Profiles of the pseudopotential $U(\sigma)$, for $H=0.5, A=0$ (solid line) and $H=0.5, A=1$ (dashed line). The fixed points are $\sigma_{0}=1.03(A=0)$ and $\sigma_{0}=1.43(A=1)$.

$$
V_{H}=-A \sigma e^{-\xi^{2} / 2 \sigma^{2}}-A \sqrt{\frac{\pi}{2}} \xi \operatorname{Erf}\left(\frac{\xi}{\sqrt{2} \sigma}\right)+\text { const }
$$

where Erf is the error function. The integration constant is chosen so that $V_{H}( \pm a)=0$, with $2 a$ being the total size of the system, and letting $a \rightarrow \infty$ at the end of the calculation. As the potential $V_{H}$ is not bounded, a divergence appears in the Lagrangian density when integrated over space. However, the divergent term does not depend on the dynamical variables $d$ and $\sigma$, so that it can be ignored.

Using the above Ansatz, one obtains the Lagrangian

$$
\begin{aligned}
L \equiv & \frac{1}{\sqrt{2 \pi} A} \int \mathcal{L} d x=\frac{\dot{d}^{2}+\dot{\sigma}^{2}}{2}-\frac{d^{2}+\sigma^{2}}{2}+\frac{\sqrt{2}}{2} A \sigma-\frac{\sqrt{3} A^{2}}{6 \bar{n}^{2} \sigma^{2}} \\
& -\frac{H^{2}}{8 \sigma^{2}},
\end{aligned}
$$

which only depends on two degrees of freedom, namely the dipole $d$ and the variance $\sigma$. The Euler-Lagrange equations corresponding to the Lagrangian $L$ read as

$$
\begin{gathered}
\ddot{d}+d=0 \\
\ddot{\sigma}+\sigma=\frac{\sqrt{2} A}{2}+\frac{\sqrt{3} A^{2}}{3 \bar{n}^{2} \sigma^{3}}+\frac{H^{2}}{4 \sigma^{3}} .
\end{gathered}
$$

The quantum-well potential $V_{\text {conf }}$ manifests itself in the harmonic forces on the left-hand side of both Eqs. (7) and (8). As expected, the equations for $d$ and $\sigma$ decouple for purely harmonic confinement. Equation (7) describes rigid oscillations of the electron gas at the effective plasmonic frequency, i.e., the Kohn mode. ${ }^{3,19}$ Equation (8) describes the dynamics of the breather mode, which features coherent oscillations of the width of the electron density. The three terms in the right-hand side of Eq. (8) correspond to the Coulomb repulsion (Hartree term), the electron pressure, and the quantum Bohm potential, respectively. The breather Eq. (8) can be written as $\ddot{\sigma}=-d U / d \sigma$, where $U(\sigma)$ is a pseudopotential defined as $U=\sigma^{2} / 2-\sqrt{2} A \sigma / 2+\sqrt{3} A^{2} /\left(6 \bar{n}^{2} \sigma^{2}\right)$ $+H^{2} /\left(8 \sigma^{2}\right)$. From the shape of the pseudopotential (Fig. 1), it 


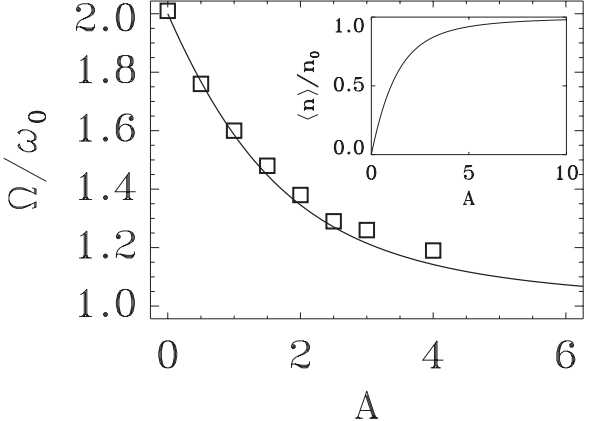

FIG. 2. The breather frequency $\Omega$ as a function of $A$, for $H$ $=0.5$. Solid line: analytical results from the Lagrangian method. Squares: the Wigner-Poisson (WP) simulations. The inset shows the mean electron density $\langle n\rangle=A / \sqrt{2} \sigma_{0}$ as a function of $A$.

follows that $\sigma$ will always execute nonlinear oscillations around a stable fixed point $\sigma_{0}(A, H)$, which is a solution of the algebraic equation $U^{\prime}\left(\sigma_{0}\right)=0$.

So far, we have not specified the value of the average density $\bar{n}$ that appears in the EOS, $P=n^{3} / \bar{n}^{2}$, written in normalized units. It is natural to assume that $\bar{n}$ takes some value smaller than the peak density at equilibrium $A / \sigma_{0}$. The correct way to compute this value is to average the square of the density using $n$ itself, i.e., $\bar{n}^{2} \equiv\left\langle n^{2}\right\rangle=\int n^{3} d x / \int n d x$ $=A^{2} /\left(\sqrt{3} \sigma_{0}^{2}\right)$. A useful check can be performed by plugging this expression into Eq. (8) and neglecting the Hartree potential, which yields the equilibrium variance $\sigma_{0}=[1+(1$ $\left.\left.+H^{2}\right)^{1 / 2}\right]^{1 / 2} / \sqrt{2}$. This expression displays the correct low- and high-temperature limits for the quantum harmonic oscillator: $\sigma_{0} \rightarrow 1$, for $H \rightarrow 0$; and $\sigma_{0} \sim \sqrt{H / 2}$, for $H \gg 1$.

With the above prescription for $\bar{n}$, the pseudopotential becomes $U=\sigma^{2} / 2-\sqrt{2} A \sigma / 2+\sigma_{0}^{2} /\left(2 \sigma^{2}\right)+H^{2} /\left(8 \sigma^{2}\right)$. The frequency $\Omega=\Omega(A, H)$ of the breather mode, corresponding to the oscillations of $\sigma$, can be obtained by linearizing the equation of motion [Eq. (8)] in the vicinity of the stable fixed point of $U(\sigma)$. The dependence of the breather frequency with $A$ (i.e., with the electron density) is shown in Fig. 2. For $A=0$ (i.e., without the Coulomb interaction) the exact frequency is $\Omega=2 \omega_{0}$. For finite $A$, the breather frequency decreases and approaches $\Omega=\omega_{0}$, for $A \rightarrow \infty$. The latter limit can be understood by noting that for large $A$ the electron density becomes flatter and flatter, due to the strong Coulomb repulsion. Thus, in the limit $A \rightarrow \infty$ we end up with a uniform electron density exactly neutralized by the ion density background. For such a homogeneous system, the Bohm-Gross dispersion relation holds, which for long wavelengths yields $\Omega=\omega_{0}$. Indeed, if one computes the average density using the prescription used for the EOS, one obtains $\langle n\rangle=\int n^{2} d x / \int n d x=A /\left(\sqrt{2} \sigma_{0}\right) \rightarrow 1$, for $A \rightarrow \infty$ (see the inset of Fig. 2). Thus, as expected, for large Coulomb effects the average electron density becomes equal to the ion background density.

\section{SIMULATIONS}

In order to check the validity of the above results, we performed numerical simulations of the Wigner-Poisson

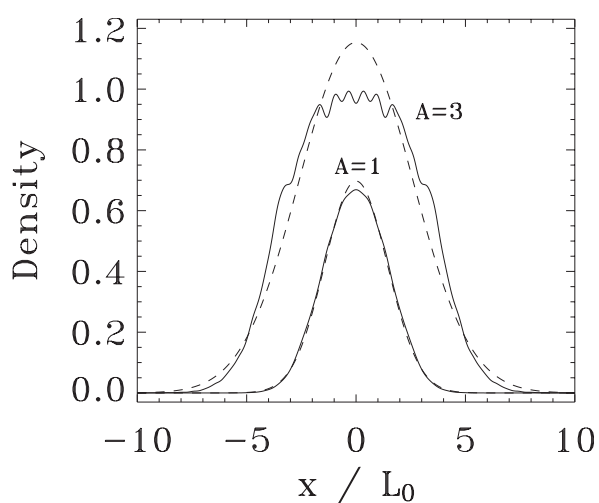

FIG. 3. Solid lines: electron density profiles at $\omega_{0} t=150$ from the WP simulations for $H=0.5$ and two values of $A$. The dashed lines represent GAUSSIAN distributions with the same width and the same area as the numerical curves.

(WP) system, which is equivalent to the time-dependent Hartree equations. ${ }^{15}$ In the normalized variables, the Wigner pseudoprobability distribution $f(x, v, t)$ satisfies the evolution equation

$$
\frac{\partial f}{\partial t}+v \frac{\partial f}{\partial x}-i \int \frac{d x^{\prime} d v^{\prime}}{2 \pi H^{2}} \delta V_{\mathrm{eff}} e^{i\left(v-v^{\prime}\right) x^{\prime} / H} f\left(x, v^{\prime}, t\right)=0,
$$

and is coupled to the Poisson equation. In Eq. (9), $\delta V_{\text {eff }}$ $\equiv V_{\text {eff }}\left(x+x^{\prime} / 2, t\right)-V_{\text {eff }}\left(x-x^{\prime} / 2, t\right)$. It is important to note that this is a microscopic quantum mean-field model, much more general than the hydrodynamic model on which our Lagrangian theory was based.

The initial condition used in the simulations is a quantum canonical distribution for the harmonic oscillator at finite temperature, ${ }^{20}$ where the spatial width $\sigma_{0}$ has been adjusted to the value obtained from the Lagrangian approach to account for the Coulomb repulsion. This is very close, but not quite identical, to an exact equilibrium of the WP equations, so that the width of the electron density starts to oscillate. We then compute the evolution of the dispersion $\left\langle x^{2}\right\rangle^{1 / 2}$ $=\left(\int f x^{2} d x d v / \int f d x d v\right)^{1 / 2}$ and its frequency spectrum, which generally shows a sharp peak at a dominant frequency.

The results of the WP simulations are plotted in Fig. 2 (squares) and agree very well with the theoretical curve based on the Lagrangian approach. The agreement slightly deteriorates for larger values of $A$, because the electron density deviates from the GAUSSIAN profile due to strong Coulomb repulsion. This is clearly visible in Fig. 3, where we represent the evolved density profiles for two values of $A$. For $A=1$, the profile is still approximately GAUSSIAN, whereas for $A=3$ an intricate internal structure has developed. Nevertheless, even in this case, the error on the frequency is still just over $3 \%$.

Table I shows that the breather frequency depends weakly on the parameter $H$ (and hence on the electron temperature). Our theoretical results are in good agreement with the simulations, except for $H=1$. For this case, the frequency spectrum is particularly broad, denoting a significant fragmentation of the resonance. 
TABLE I. The breather frequency $\Omega$ and the equilibrium width $\sigma_{0}$ for $A=1$ and for various values of $H$.

\begin{tabular}{cccc}
\hline \hline$H$ & $\sigma_{0}$ & $\Omega$ (theory) & $\Omega($ sim. $)$ \\
\hline 0.00 & 1.41 & 1.58 & 1.60 \\
0.50 & 1.43 & 1.59 & 1.60 \\
1.00 & 1.47 & 1.60 & 1.51 \\
1.50 & 1.52 & 1.61 & 1.57 \\
2.00 & 1.59 & 1.63 & 1.63 \\
3.00 & 1.73 & 1.66 & 1.70 \\
\hline \hline
\end{tabular}

\section{NONPARABOLIC WELLS}

For a parabolic potential well, there is no coupling between the breather and dipole modes, which may render the experimental detection of the breather mode by optical means difficult to realize in practice.

It can be shown that nonparabolic corrections do not introduce any linear coupling if the confining potential is symmetric. A more interesting situation arises for asymmetric wells, ${ }^{21}$ which we model by adding a small cubic term to the confining potential, $V_{\text {cub }}(x)=(K / 3) x^{3}$. The equations of motion are then

$$
\begin{gathered}
\ddot{d}+d=-K\left(\sigma^{2}+d^{2}\right), \\
\ddot{\sigma}+\sigma=\frac{\sqrt{2} A}{2}+\frac{\sqrt{3} A^{2}}{3 \bar{n}^{2} \sigma^{3}}+\frac{H^{2}}{4 \sigma^{3}}-2 K \sigma d .
\end{gathered}
$$

Linearizing Eqs. (10) and (11) around the stable fixed point $\left(d_{0}, \sigma_{0}\right)$, we indeed find a coupling between the breather and the dipole, with resonant frequencies $\Omega_{ \pm}$.

We model the coupling to the laser field by instantaneously shifting the initial dipole of a small quantity, i.e.,

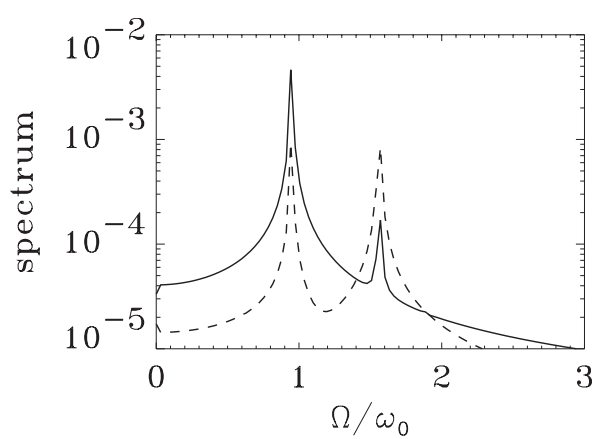

FIG. 4. The frequency spectra of the dipole (solid line) and breather (dashed) modes, for an asymmetric well with $A=1, H$ $=0.5$, and $K=-0.1$.

$d(0)=d_{0}+\tilde{d}$. Figure 4 shows a typical spectrum obtained from the numerical solution of Eqs. (10) and (11), which is proportional to the optical absorption spectrum commonly measured in the experiments. The sharp peaks correspond to the resonant frequencies $\Omega_{-}=0.97$ and $\Omega_{+}=1.58$, which are rather close to those obtained for parabolic confinement.

The breather mode can thus be triggered using a purely dipolar excitation, and a clear signature of the breather frequency can be observed in the optical absorption spectrum. This opens the way to optically detecting the breather mode by means of standard pump-probe experiments. Finally, the methods used here could be readily extended to threedimensional nanostructures, and may find applications in related areas such as quantum free-electron lasers. ${ }^{22}$

\section{ACKNOWLEDGMENTS}

This work was partially supported by the Alexander von Humboldt Foundation and by the Agence Nationale de la Recherche.
${ }^{1}$ P. Zoller et al., Eur. Phys. J. D 36, 203 (2005).

${ }^{2}$ J. N. Heyman et al., Appl. Phys. Lett. 72, 644 (1998).

${ }^{3}$ W. Kohn, Phys. Rev. 123, 1242 (1961).

${ }^{4}$ M. N. Harakeh, K. van der Borg, T. Ishimatsu, H. P. Morsch, A. van der Woude, and F. E. Bertrand, Phys. Rev. Lett. 38, 676 (1977).

${ }^{5}$ D. H. Youngblood, C. M. Rozsa, J. M. Moss, D. R. Brown, and J. D. Bronson, Phys. Rev. Lett. 39, 1188 (1977).

${ }^{6}$ K. Góral, M. Brewczyk, and K. Rzazewski, Phys. Rev. A 67, 025601 (2003).

${ }^{7}$ Y. Zhou and G. Huang, Phys. Rev. A 75, 023611 (2007).

${ }^{8}$ H. Portales et al., J. Chem. Phys. 115, 3444 (2001).

${ }^{9}$ M. Santer, B. Mehlig, and M. Moseler, Phys. Rev. Lett. 89, 286801 (2002).

${ }^{10}$ H. O. Wijewardane and C. A. Ullrich, Appl. Phys. Lett. 84, 3984 (2004).

${ }^{11}$ G. Manfredi and F. Haas, Phys. Rev. B 64, 075316 (2001).

${ }^{12}$ G. Manfredi, Fields Inst. Commun. 46, 263 (2005).

${ }^{13}$ N. Crouseilles, P. A. Hervieux, and G. Manfredi, Phys. Rev. B
78, 155412 (2008).

${ }^{14}$ C. L. Gardner and C. Ringhofer, Phys. Rev. E 53, 157 (1996).

${ }^{15}$ G. Manfredi and P.-A. Hervieux, Appl. Phys. Lett. 91, 061108 (2007).

${ }^{16}$ G. M. Gusev, A. A. Quivy, T. E. Lamas, J. R. Leite, A. K. Bakarov, A. I. Toropov, O. Estibals, and J. C. Portal, Phys. Rev. B 65, 205316 (2002).

${ }^{17}$ J. N. Heyman, K. Unterrainer, K. Craig, B. Galdrikian, M. S. Sherwin, K. Campman, P. F. Hopkins, and A. C. Gossard, Phys. Rev. Lett. 74, 2682 (1995).

${ }^{18}$ M. Matuszewski, E. Infeld, B. A. Malomed, and M. Trippenbach, Phys. Rev. Lett. 95, 050403 (2005).

${ }^{19}$ J. F. Dobson, Phys. Rev. Lett. 73, 2244 (1994).

${ }^{20}$ K. Imre et al., J. Math. Phys. 8, 1097 (1967).

${ }^{21}$ E. Rosencher and P. Bois, Phys. Rev. B 44, 11315 (1991); E. Dupont, et al., IEEE J. Quantum Electron. 42, 1157 (2006).

${ }^{22}$ N. Piovella, M. M. Cola, L. Volpe, A. Schiavi, and R. Bonifacio, Phys. Rev. Lett. 100, 044801 (2008). 